\title{
A Non-Invasive Assessment of Ground Reaction Forces in the Human Leg in Response to Walking, Jogging, Running and Jumping
}

\author{
Jessica Pingel ${ }^{1 *}$, Adrian Harrison ${ }^{2}$ \\ ${ }^{1}$ Department of Neuroscience, Faculty of Health \& Medical Sciences, University of Copenhagen, København, Denmark \\ ${ }^{2}$ PAS, Department of Physiology, Faculty of Health \& Medical Sciences, University of Copenhagen, Frederiksberg, Denmark \\ Email: *jpingel@sund.ku.dk
}

How to cite this paper: Pingel, J. and Harrison, A. (2020) A Non-Invasive Assessment of Ground Reaction Forces in the Human Leg in Response to Walking, Jogging, Running and Jumping. Open Journal of Orthopedics, 10, 152-160.

https://doi.org/10.4236/ojo.2020.107017

Received: June 22, 2020

Accepted: July 26, 2020

Published: July 29, 2020

Copyright () 2020 by author(s) and Scientific Research Publishing Inc. This work is licensed under the Creative Commons Attribution International License (CC BY 4.0).

http://creativecommons.org/licenses/by/4.0/

\begin{abstract}
Running is one of the most popular forms of exercise. Even though regular exercise is beneficial to human health, running is also often associated with an increased risk of injury. Lack of shock absorption in running shoes has often been stated as one of the main reasons for why running-related injuries have been on the increase. The aim of the present study was to assess the degree to which ground reaction forces (GRF) can be dissipated in the human leg in a barefoot subject, in connection with diverse physical activities. Acoustic myography (AMG), a non-invasive technique that records pressure waves from contracting muscles as well as the harmonic damping of ligaments, was applied to four anatomical sites on the subject's leg, during barefoot walking, jogging, running and jumping. The data for walking on a hard surface show much lower ESTi ${ }^{\mathrm{TM}}$ parameters than those for the soft surface, and these lower values are observed mainly for sites 1 (toes; 65\%) and 2 (ankle; 53\%), respectively. AMG parameters for jogging reveal this gait to have very low ES$\mathrm{Ti}^{\mathrm{TM}}$ parameters for site 1 and site 2 (ESTi 2 - 3), yet similar for both surfaces. The data for running on a hard and soft surface revealed much lower $\mathrm{ESTi}^{\mathrm{TM}}$ parameters (38\%) than those for sites 3 (knee) and 4 (hip). The data from the big jump, reveal that on a hard surface, the lowest $\mathrm{ESTi}^{\mathrm{TM}}$ parameters were for sites 1 (toes; 46\%) and 2 (ankle; 27\%), compared to values on a soft surface. The speed with which GRFs were transmitted up the leg varied from site to site and also with the type of activity, ranging from undetectable to approx. $60 \mathrm{~m} / \mathrm{sec}$. The present study reveals that the ankle joint is exposed to the greatest forces during jumping and running. In addition, this study has confirmed that exercising on a hard surface does indeed increase the stress forces on the toes and ankles. It is interesting to note that the data reveal that toes and ankles absorb most of the GRF during running, while the knee and hip
\end{abstract}


joint remain unaffected, although a more detailed study involving a larger number of subjects and shoe types is now needed.

\section{Keywords}

Muscle, Activity, Acoustic Myography

\section{Introduction}

It is known that leg stiffness is constantly assessed and adjusted during periods of physical activity [1]. Indeed, muscles in the foot and around the ankle are known to play an important role in support, postural balance and dynamic stability [2].

Whilst mechanical stress in the foot and lower limb can be alleviated by use of correct footwear [3], others have suggested that footwear per se may in itself reduce the activity patterns of supportive muscles compared with a barefoot state [4].

In addition to the type of footwear a subject selects, it is also known that step parameters and kinematic measurements can be affected by the type of surface an individual exercises on [5]. Ferris and coworkers [1] showed that human runners adjust the stiffness of their supportive leg during steady state running in accordance with the type of surface on which they are exercising. Other studies are less clear cut and tend to contradict each other with regards to lower extremity biomechanics when running on different surfaces [6]. For example, Tessutti and colleagues [7] reported that running on hard surfaces such as asphalt or concrete increases the peak pressure and decreases the contact time of a runner's limb compared to running on grass. These authors went on the conclude that running on a grass surface with very little stiffness, may serve to allow the body to better attenuate forces by increasing contact time and decreasing stride length [8]. In a study of 15 recreational runners aged approx. 20 years ( 7 men; 8 women), measurements made during both fast and slow track runs revealed that the pronation excursion, the braking and the impact were significantly greater when compared to values from the same individuals whilst undertaking a grass run [6].

The aim of this study therefore was: 1) to assess the suitability of acoustic myography as a technique for assessing how ground reaction forces are dealt with in key sites up the human lower limb, and 2) to determine differences between these key anatomical sites with gaits of increasing intensity.

\section{Materials and Methods}

\section{Ethical Approval}

The method applied was non-invasive, and the study followed the guidelines set by the Helsinki Declaration 2013

(https://www.wma.net/policies-post/wma-declaration-of-helsinki-ethical-princi 
ples-for-medical-research-involving-human-subjects/).

\section{Subject}

One healthy and trained subject participated in this study, with the following details: gender female, age39 years, weight $60 \mathrm{~kg}$, BMI 22.3 .

\section{Activities}

The subject was measured whilst physically active and engaged in a number of diverse gaits without shoes, which included, walking, jogging, running and a big jump on the spot. These diverse gaits were measured for both a hard and smooth concrete floor, as well as for a soft grass lawn, with an irregular surface. The order of measurement was as follows: walking without shoes on a hard surface, jogging on a hard surface without shoes, running on a hard surface without shoes, a big on the spot jump without shoes, after which all of these were repeated on a soft surface.

\section{Recordings}

Acoustic myography (AMG) is a biomechanical method measuring generated pressure waves from a contracting muscle [9] [10]. AMG recordings were carried out with a CURO unit and CURO sensors (CURO-Diagnostics ApS, Denmark; formerly MyoDynamikApS) and followed in real time on an iPADAir (Apple Inc., Cupertino, CA, USA) via the App "CURO Clinic" and a specialized data recording system. This allowed us to see the actual wave recordings and the ESTi score while recording. We used $50 \mathrm{~mm}$ sensors with a frequency recording range of $(0.5-20) \pm 0.5 \mathrm{kHz}$, and the sampling rate was $4 \mathrm{kHz}$. Recorded data was stored to the CURO Unit and after completion of measurements transferred to the CURO software (https://app.myodynamik.com). The ESTi-score with its three components: 1) efficiency (E-score) 2) temporal fibre recruitment (T-score) and 3) spatial fibre recruitment (S-score), was calculated using the company software [9] [10].

As an example, the $\mathrm{S}$-score was determined as the signal amplitude in relation to a full $6 \mathrm{~dB}$ signal (measured as approx. $1 \mathrm{~V}$ ). For more intuitive assessment of optimal muscle function, a scale of 0 - 10 was adopted, where 0 was considered as $0 \%$ optimal and 10 was considered $100 \%$ optimal. To calculate the score, the measured $\mathrm{mV}$ amplitude was subtracted from the maximal $\mathrm{mV}$ amplitude that could be accurately detected. The difference was then divided by the maximal amplitude and multiplied by 10 to yield a $0-10$ scoring system. By way of an example, an S-score of 8 represents a signal with a very small amplitude (approx. $0.3 \mathrm{~V}$ ), whereas an S-score of 1 represents a relatively large amplitude signal (approx. $0.7 \mathrm{~V}$ ). The T- and E-score was calculated in a similar way to their full signal to give a score scale from $0-10$, where 0 is full activity and 10 is no activity.

\section{Measurement locations}

All measurements were taken from the left leg, and without shoes. A $20 \mathrm{~mm}$ sensors (CURO-Diagnostics ApS, Denmark) in connection with a CURO unit, was used for AMG measurements which were carried out at the level of the big toe (the joint between $1^{\text {st }}$ proximal phalanx and the $1^{\text {st }}$ metatarsal; 1 ), the ankle (medial malleolus; 2), the lower leg (tibial tuberosity; 3 ) and the hip (iliac crest; 
4). Site 2 was $12 \mathrm{~cm}$ away from site 1 and $5 \mathrm{~cm}$ above the ground, site 3 was 38 $\mathrm{cm}$ away from site 1 , and site 4 was $93 \mathrm{~cm}$ away from site 1 .

\section{Statistical Analysis}

Owing to the fact that the data were collected from just one subject, no statistical tests were performed. Differences are expressed as percentage change either compared with a soft surface, or between recording sites.

\section{Results}

\section{AMG parameters}

The data obtained whilst walking on the hard surface show much lower E, S and $\mathrm{T}$ parameters than those for the soft surface, and these lower values are observed mainly for sites 1 and 2 (see Figure 1(a)). Interestingly, when walking on your toes on either a hard or a soft surface, the $S$ and $T$ scores for site 1 greatly improve, but worsen for site 2 , and remain optimal for sites 3 and 4 .

AMG parameters measured during jogging reveal very low $\mathrm{E}, \mathrm{S}$ and $\mathrm{T}$ scores for site 1 and site 2 whether the subject is jogging on a hard or a soft surface (see Figure 1(b)).

Recordings made from the subject when running revealed that most of the GRF is being absorbed at site 2, that site 1 is very comparable to the values obtained for walking on a flat hard surface (see Figure 1(c)).

Data for the big jump reveal that on a hard surface, the lowest $\mathrm{E}, \mathrm{S}$ and $\mathrm{T}$ scores were for site 2 , and that on a soft surface the values at site 2 were greatly improved and similar to those for site 1 (see Figure $1(\mathrm{~d})$ ).

GRF transmission velocity

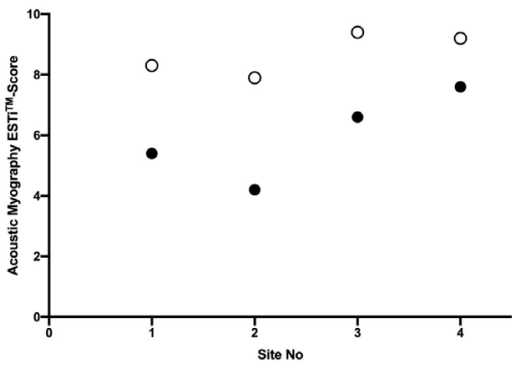

(a)

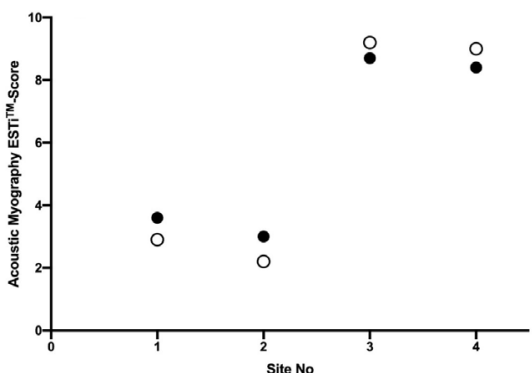

(c)

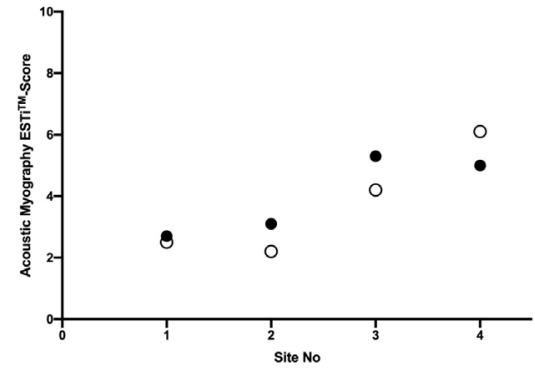

(b)

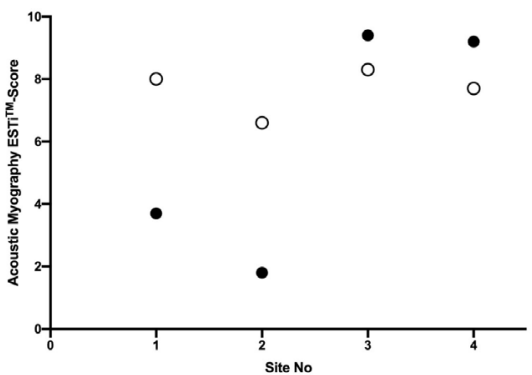

(d)

Figure 1. An overview of the combined $\mathrm{ESTi}^{\mathrm{TM}}$-score for the AMG signals at sites 1,2, 3 and 4, for (a) Walking, (b) Jogging, (c) Running and (d) Big jump, for a subject on a hard - and a soft surface $\bigcirc$. 
The speed of transmission of the GRF through the left leg was found to range from undetectable to having a speed of $13-42 \mathrm{~m} / \mathrm{sec}$ at site 2, depending on the type of activity (see Figure 2). The speed of transmission of the GRF through the left leg was found to range from undetectable to having a speed of $20-66 \mathrm{~m} / \mathrm{sec}$ at site 3, depending on the type of activity. The speed of transmission of the GRF through the left leg was found to range from undetectable to having a speed of $18-62 \mathrm{~m} / \mathrm{sec}$ at site 4 , depending on the type of activity.

In terms of walking, the speed of transmission of the GRF was found to be 3.4 - $3.5 \mathrm{~m} / \mathrm{sec}$ on a hard surface, to $1.8-2.2 \mathrm{~m} / \mathrm{sec}$ on a soft surface.

\section{Discussion}

The present study has shown that acoustic myography as a technique, is quite capable of assessing the signal strength and the transfer of ground reaction forces through key sites in the human lower limb. Moreover, using this technique we have been able to determine signal differences between four key anatomical sites, when comparing gaits of increasing intensity.

The data presented in Figure 1 reveal that in general there are higher ES$\mathrm{Ti}^{\mathrm{TM}}$-scores for sites 3 and 4, rather than 1 and 2. This means that a higher ES$\mathrm{Ti}^{\mathrm{TM}}$-score represents a small amplitude signal, in other words, it demonstrates that the GRF signal has been adequately damped by the time it reaches the knee and the hip (sites $3 \& 4$, respectively). The data also reveal that the signal is often worst (lower $\mathrm{ESTi}^{\mathrm{TM}}$-score) for site 2 (ankle), and particularly when recorded on a hard surface. This is particularly interesting since it has been published that foot and ankle injuries in sport remains very common with incidences of approx. 35 in association football, 14 in rugby and 14 in American football per 1000 people per hour [11].

Of interest in this study, is the finding that a comparison of barefoot walking clearly poses less of an impact on the foot and ankle when performed on a soft surface compared with a hard surface. Barefoot running has become popular since it has been associated with an increased energy storage in the runner's arch [12]. Moreover, it has been concluded that barefoot running does not increase the relative injury rate of runners, compared with those wearing running shoes

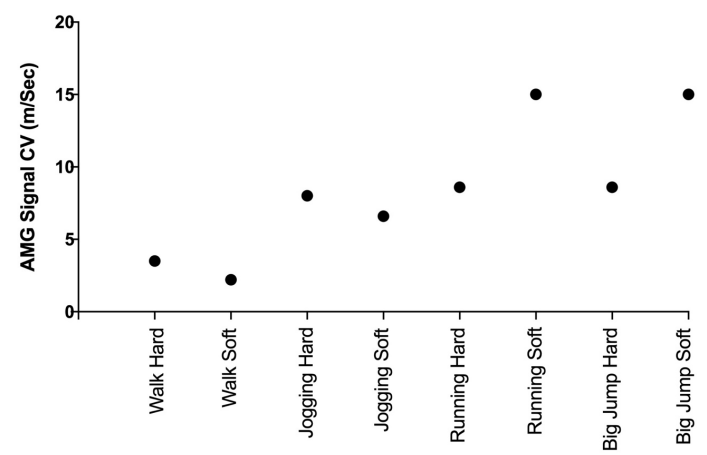

Figure 2. A plot of the conduction velocities for the AMG signals between sites 1 and 2 for different gaits on both a hard and a soft surface. 
[13]. However, in contrast, another study reported that there is an increased muscle activation and impact acceleration in the tibia of barefoot runners, which when combined highlights a potential risk of injury compared to those running with shoes [14].

In a recent study, is was stated that training shoes, which have a thick and well-cushioned midsole, are the most common type of running shoe [15]. This is of interest, since a study of racing flats, which are thought to have less cushioning, documented that plantar pressures and associated forces are greater for racing flats than for training shoes [16]. These authors went on to conclude that racing flats are associated with a higher risk of stress fracture of the metatarsal bones compared to training shoes [16]. However, in another study it was reported that whilst shoe construction changes mechanical demands whilst running, runners quickly become habituated to the demands of a given shoe [17].

In Figure 2 we show that the conduction velocity of the AMG signal increases in a more or less linear fashion with the level of intensity of physical activity: walking to jogging to running to jumping. This study reveals the clear increase in the speed with which the GRF signal is transmitted from the point of impact up through the limb from a relatively slow rate $(3.4-3.5 \mathrm{~m} / \mathrm{sec}$ on a hard surface $/ 1.8-2.2 \mathrm{~m} / \mathrm{sec}$ on a soft surface) when walking, to a much faster rate (40 $60 \mathrm{~m} / \mathrm{sec}$ on a soft surface) when running or jumping on the spot. In a study of runners with a prosthetic running blade it was found that changing the elastic properties of the blade itself had an impact on the runners approach angle and their ground reaction force, but interestingly, it did not affect their limb stiffness, which was comparable with that of healthy controls [18]. This observation is most likely the result of pre-tensioning within the muscles and ligaments of the limb during relatively high-speed activities, and is supported by the finding that running speed is linearly associated with limb stiffness [19]. Mauroy and colleagues [20], measuring blade prosthesis properties in transfemoral amputees also showed that not only does the stiffness of a prosthetic blade change with increasing running speed, but that the stiffness of the limb muscles (hip muscles) increased too.

The present studies observation of conduction velocity differences with gait and physical intensity is supported by a study of the use of flip-flops, which showed that wearers had a significantly slower walking speed, a higher ankle and sub-tarsal joint range of motion and higher shear ankle joint contact forces than individuals wearing sports shoes [21]. Thus, the use of flip-flops, forcing the wearer to move at a slower speed, might be expected to result in a relatively less stiff foot and ankle, a slower conduction velocity and a lower GRF, all of which might be expected to reduce the incidence of training injury. Indeed, it has been estimated that for runners covering between 50 and 70 miles a week, there is a $50 \%$ chance of knee injury due to repetitive loading of the joint, furthermore it is mentioned that the material used to construct the midsole of runners shoes is the limiting factor in terms of shoe performance, and that this must be improved in order to offer runners a more long-term form of protection [22]. It should be 
noted, however, that running shoes built with extreme cushioning and an oversized midsole (referred to as maximalist shoes) have not been found to lower the external impact loading when compared to traditional running shoes [23]. There is now a great need for further studies using AMG with a larger sample size, as well as different shoe and surface types.

\section{Conclusion}

This preliminary study suggests that barefoot walking is not a very comprehensive gait for the ankle but is better than both jogging and running for the toes when a subject is walking on a soft surface. Jogging results in a low $\mathrm{ESTi}^{\mathrm{TM}}$-score for all 4 measured sites compared with walking, even on a hard surface, and should therefore be seen as a relatively high impact gait for someone who is barefoot. The same can be said for running, although in contrast with jogging, this gait apparently has less impact on the knee and hip joints. Finally, anything involving a big jump on the spot on a hard surface, clearly has a huge impact on the ankle.

\section{Author's Contributions}

All authors have contributed equally to this work and have approved the final version of the manuscript. All authors are designated as authors and are qualified for authorship, and are all listed as authors.

\section{Acknowledgements}

The authors are not aware of anyone that needs to be acknowledged for their help with this work.

\section{Conflicts of Interest}

$\mathrm{AH}$ is currently trying to establish a company to produce the acoustic myography units. The authors declare no conflict of interest.

\section{References}

[1] Ferris, D.P., Liang, K. and Farley, C.T. (1999) Runners Adjust Leg Stiffness for Their First Step on a New Running Surface. Journal of Biomechanics, 32, 787-794. https://doi.org/10.1016/S0021-9290(99)00078-0

[2] Honeine, J.-L., Schieppati, M., Gagey, O. and Do, M.-C. (2013) The Functional Role of the Triceps Surae Muscle during Human Locomotion. PLOS ONE, 8, e52943. https://doi.org/10.1371/journal.pone.0052943

[3] Taunton, J.E., Ryan, M.B., Clement, D.B., McKenzie, D.C., Lloyd-Smith, D.R. and Zumbo, B.D. (2003) A Prospective Study of Running Injuries: The Vancouver Sun Run “In Training” Clinics. British Journal of Sports Medicine, 37, 239-244. https://doi.org/10.1136/bjsm.37.3.239

[4] Roca-Dols, A., Losa-Iglesias, M.E., Sánchez-Gómez, R., López-López, D., Becerro-de-Bengoa-Vallejo, R. and Calvo-Lobo, C. (2018) Electromyography Comparison of the Effects of Various Footwear in the Activity Patterns of the Peroneus Longus and Brevis Muscles. Journal of the Mechanical Behavior of Biomedical Ma- 
terials, 82, 126-132. https://doi.org/10.1016/j.jmbbm.2018.03.003

[5] Gates, D.H., Wilken, J.M., Scott, S.J., Sinitski, E.H. and Dingwell, J.B. (2012) Kinematic Strategies for Walking across a Destabilizing Rock Surface. Gait \& Posture, 35, 36-42. https://doi.org/10.1016/j.gaitpost.2011.08.001

[6] Hollis, C.R., Koldenhoven, R.M., Resch, J.E. and Hertel, J. (2019) Running Biomechanics as Measured by Wearable Sensors: Effects of Speed and Surface. Sports Biomechanics, 1-11. https://doi.org/10.1080/14763141.2019.1579366

[7] Tessutti, V., Trombini-Souza, F., Ribeiro, A.P., Nunes, A.L. and Sacco, I.C. (2010) In-Shoe Planta Pressure Distribution during Running on Natural Grass and Asphalt in Recreational Runners. Journal of Science \& Medicine in Sport, 13, 151-155. https://doi.org/10.1016/j.jsams.2008.07.008

[8] Tessutti, V., Ribeiro, A.P., Trombini-Souza, F. and Sacco, I.C. (2012) Attenuation of Foot Pressure during Running on Four Different Surfaces: Asphalt, Concrete, Rubber, and Natural Grass. Journal of Sports Sciences, 30, 1545-1550. https://doi.org/10.1080/02640414.2012.713975

[9] Harrison, A.P. (2018) A More Precise, Repeatable and Diagnostic Alternative to Surface Electromyography-An Appraisal of the Clinical Utility of Acoustic Myography. Clinical Physiology and Functional Imaging, 38, 312-325. https://doi.org/10.1111/cpf.12417

[10] Claudel, C.G., Ahmed, W., Elbrond, V.S., Harrison, A.P. and Bartels, E.M. (2018) The Relation between Maximal Voluntary Force in M. Palmaris Longus and the Temporal and Spatial Summation of Muscle Fiber Recruitment in Human Subjects. Physiological Reports, 6, e13580. https://doi.org/10.14814/phy2.13580

[11] Fong, D.T.P., Hong, Y., Chan, L.K., Yung, P.S.H. and Chan, K.M. (2007) A Systematic Review on Ankle Injury and Ankle Sprain in Sports. Sport Medicine, 37, 73-94. https://doi.org/10.2165/00007256-200737010-00006

[12] Altman, A.R. and Davis, I.S. (2012) Barefoot Running: Biomechanics and Implications for Running Injuries. Current Sports Medicine Reports, 11, 244-250. https://doi.org/10.1249/JSR.0b013e31826c9bb9

[13] Hollander, K., Heidt, C., Van Der Zwaard, B.C., Braumann, K.M. and Zech, A. (2017) Long-Term Effects of Habitual Barefoot Running and Walking: A Systematic Review. Medicine and Science in Sports \& Exercise, 49, 752-762. https://doi.org/10.1249/MSS.0000000000001141

[14] Lucas-Cuevas, A.G., Priego Quesada, J.L., Giménez, J.V., Aparicio, I., Jimenez-Perez, I. and Pérez-Soriano, P. (2016) Initiating Running Barefoot: Effects on Muscle Activation and Impact Accelerations in Habitually Rearfoot Shod Runners. European Journal of Sports Science, 16, 1145-1152. https://doi.org/10.1080/17461391.2016.1197317

[15] Hashizume, S., Murai, A., Hobara, H., Kobayashi, Y., Tada, M. and Mochimaru, M. (2017) Training Shoes Do Not Decrease the Negative Work of the Lower Extremity Joints. International Journal of Sports Medicine, 38, 921-927. https://doi.org/10.1055/s-0043-117412

[16] Wiegerinck, J.L., Boyd, J., Yoder, J.C., Abbey, A.N., Nunley, J.A. and Queen, R.M. (2009) Differences in Plantar Loading between Training Shoes and Racing Flats at a Self-Selected Running Speed. Gait and Posture, 29, 514-519. https://doi.org/10.1016/j.gaitpost.2008.12.001

[17] Tam, N., Darragh, I.A.J., Divekar, N.V. and Lamberts, R.P. (2017) Habitual Minimalist Shod Running Biomechanics and the Acute Response to Running Barefoot. International Journal of Sports Medicine, 38, 770-775. 
https://doi.org/10.1055/s-0043-114863

[18] Haber, C.K., Ritchie, L.J. and Strike, S.C. (2018) Dynamic Elastic Response Prostheses Alter Approach Angles and Ground Reaction Forces But Not Leg Stiffness during a Start-Stop Task. Human Movement Science, 58, 337-346.

https://doi.org/10.1016/j.humov.2017.12.007

[19] Beck, O.N., Taboga, P. and Grabowski, A.M. (2017) Reduced Prosthetic Stiffness Lowers the Metabolic Cost of Running for Athletes with Bilateral Transtibial Amputations. Journal of Applied Physiology, 122, 976-984.

https://doi.org/10.1152/japplphysiol.00587.2016

[20] Mauroy, G., De Jaeger, D., Vanmarsenille, J.-M. and Willems, P.A. (2012) The Bouncing Mechanism of Running in a Transfemoral Amputee Wearing a Blade Prosthesis. Computer Methods in Biomechanics and Biomedical Engineering, 15, 357-359. https://doi.org/10.1080/10255842.2012.713721

[21] Chen, T.L.-W., Wong, D.W.-C., Xu, Z., Tan, Q., Wang, Y., Luximon, A. and Zhang, M. (2018) Lower Limb Muscle Co-Contraction and Joint Loading of Flip-Flops Walking in Male Wearers. PLoS ONE, 13, e0193653.

https://doi.org/10.1371/journal.pone.0193653

[22] Cook, S.D., Kester, M.A. and Brunet, M.E. (1985) Shock Absorption Characteristics of Running Shoes. The American Journal of Sports Medicine, 13, 248-253. https://doi.org/10.1177/036354658501300406

[23] Chan, Z.Y.S., Au, I.P.H., Lau, F.O.Y., Ching, E.C.K., Zhang, J.H. and Cheung, R.T.H. (2018) Does Maximalist Footwear Lower Impact Loading during Level Ground and Downhill Running? European Journal of Sport Science, 18, 1083-1089. https://doi.org/10.1080/17461391.2018.1472298 